\title{
Synthesis of 3-(Arylmethylene)-1,5-benzodiazepin-2-ones from Baylis-Hillman Acetates
}

\author{
Jeong Mi Kim, Ka Young Lee, and Jae Nyoung Kim* \\ Depantment of Chemisty and Institule of Basic Science, Chonnam National Universitv, Kwangin 500-757, Korea \\ Received Juthe +, 2002
}

Key words : 1,5-Benzodiazepine, Baylis-H lillman acetate, Benzimidazole

Seven-membered heterocyeles with two heteroatoms in a 1,4-relationship are known to possess many biological activities. Particularly, aryl-annelated $\mid 1,4]$ diazepine and $\mid 1,4$ |oxazepine are crucial moiclies in many psychoactive phamaceuticals. ${ }^{1.2}$ 6-Benzylidene-oxazepane-5,7-dione is known as a valuable chiral intermediate. ${ }^{3}$ Arylmethylene benzodiazepinones have been used for the synthesis of pesticidal pyrazolobenzodiazepines and thiazinobenzodiazepines. Besides of these papers, numerous reports have been reported regarding the synthesis or biological activity of benzodiazepines' or dibenzodiazepines. ${ }^{2}$ Recently, Reiser et al. have reported combinatorial liquid-phase synthesis of | 1,4 |oxazepin-7-ones via the Baylis-f [illman reaction."

In these respects, we intended to prepare some 3-(arylmethylene)-1,5-benzodiazepin-2-one derivatives from the Baylis-l Jillman acetates. The reaction of the Baylis-I Jillman acctate $\mathbf{1 a}$ and 1,2-phenylenediamine (2) in acctonitrile in the presence of potassium carbonate gave the allylic substitution product $3 \mathbf{a}^{6}$ (Scheme 1). The $E$ and $Z$-form of 3 a could be separated easily. I leating of pure $3 \mathbf{a}-E$ in acetic acid aflorded a mixture of $\mathbf{4} \mathbf{a}^{6}$ and $\mathbf{5} \mathbf{a}^{6}$ (Scheme 2). The yield of desired 3-(benzylidene)-1,5-benzodiazepin-2-one (4a) was moderate $(36 \%)$. Instead, the benzimidazole-substituted compound 5 a was isolated in $34 \%$ yield.

To improve the yield of the desired benzodiazepine

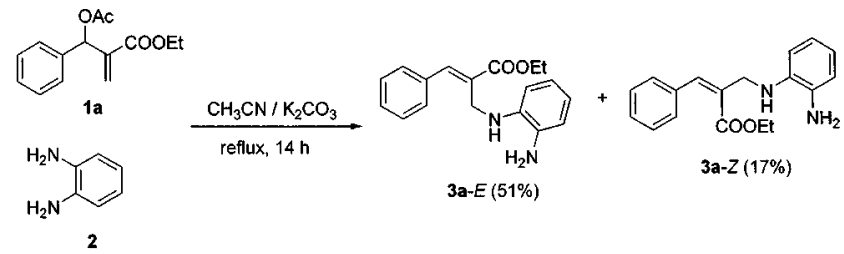

Scheme 1

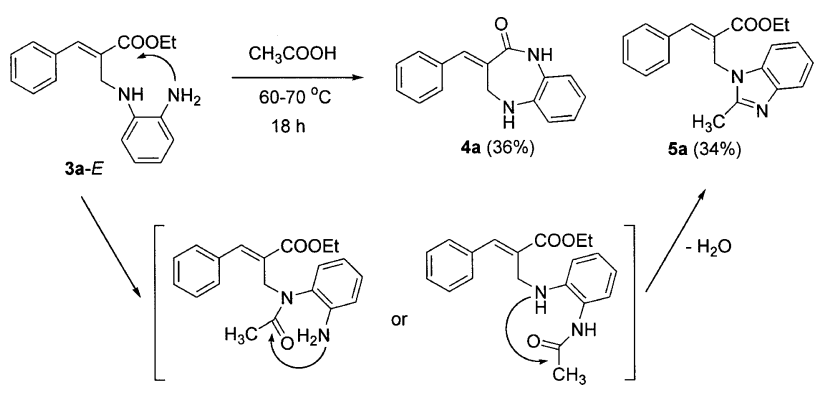

Scheme 2

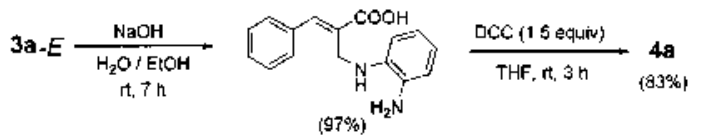

Scheme 3

derivative 4a. we examined other carboxylic acid solvent such as propionic acid. formic acid and trilluoroacetic acid as shown in Table I. Ilowever, we could not improve the yicld of 4a. In all cases, except for formic acid, differently substituted benzimidazole-substituted derivatives, $5 \mathbf{b}$ and 5c, were isolated in variable yields. It is interesting to note that the use of formic acid gave neither the corresponding benzodiazepine nor benzimidazole derivatives. Instead, di-

Table 1. Synthesis of 3-benzylidene-1.5-benzodiazepin-2-one derivalives

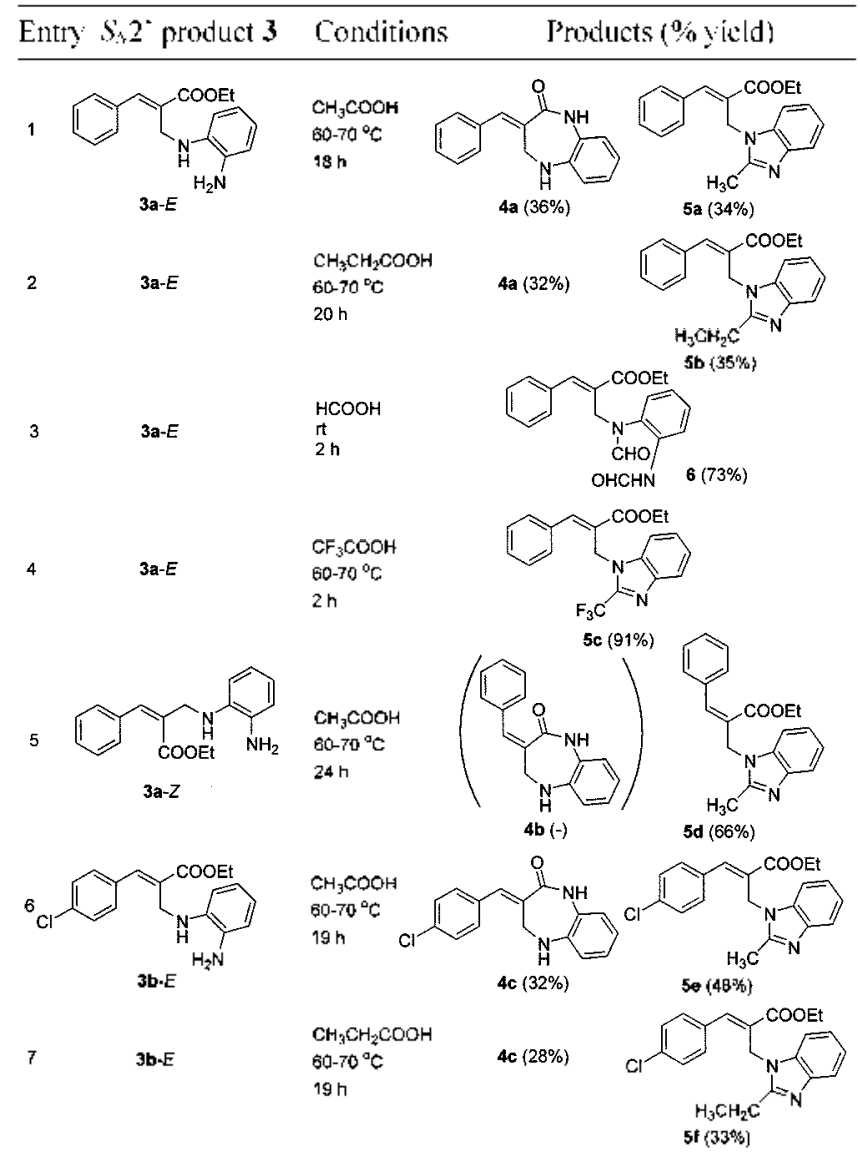


formyl derivative 6 was fommed in good yield. In fomic acid A-formy lation proceeded easily at the two nitrogen atoms. thus preventing the next cyclization toward benzodiazepine or benzimidazole.

The reaction of acetic acid and $Z$-form of $3 \mathbf{a}$ gave the benzimidazole derivative $\mathbf{5} \mathbf{d}$ as the sole product (66\%. entry 5). We could not isolate the corresponding belzodiazepine compound tb at all. We could not explain the reason at this stage. The reaction of $\mathbf{3 b}-E$ in acetic acid or in propionic acid gave the similar results (entries 6 and 7 ).

limproved synthesis of benzodiazepine derivative ta was finally carried out by using 1.3-dicyclohexylcarbodimide (DCC) method for the amide bond fomation. Hydrolysis of 3a-t: with sodium hydroxide gave the corresponding acid derivative in $97 \%$ yield. Formation of the amide bond by using DCC in THF (rt, 3h) afforded ta in $83 \%$ yield (Scheme 3).

Acknowledgment. This work was supported by the grant (No. R05-2000-000-00074-0) from the Basic Research Program of the Korea Science \& Engineering Foundation.

\section{References and Notes}

I. (a) J ce, J: Gauthice, D.: Rivero, R. A. J. Org. Chem. 1999, 64. 3060. (b) Kraus. G. A.: Liu. P. Tetrohedron Lett. 1995. 36.7595.

2. (a) Levy O.: Erez. M.: Varon. D.: Keinan. E. Bioorg . Hed (hem. I.ett. 2001. 11. 2921. (b) Liao. Y.: Venhuis. B. J.: Rodenhuis. N.: Timmeman, W: Wikstrom, II. Mejer, F.: Bartoszitk, G. D.: Botteher. II.: Serfired, C. A.: Sundel], S. J. Hed. Chem 1999. 42. 2235. (c) Colnen, V. I.: Jin, B.: Cohen, F.. I: 7 ceberg. B. R. J. Heteroctclic Chem, 1998. 35.675. (d) Liao. Y: DeBoer. I?: Meier. E.: Wikstrom. H. I. Med Chem. 1997. 40. 4146 . (e) Corles. E. C.: Islas. l? M.: (Garcia. M. M.: Romero. M. O. Z. J. Hetemochclic Chem. 1996, 33, 1723. (f) 7.hang. J..-11.: Meicr, W.: Wats. T...
Costello. I. D.: Ma. P': Ensinger. C. L.: Rodgers. I. M.: Iacobson. 1. C.: Rajagopalan. P. Tetrohedron Lett 1995.36 .8387$.

3. lietce. L. f.: Brand. S.: Pleilter. l.: Antel. l.: Hanns. K.: Sheldrick. G. M. J. fm. Chm. Soc. 1987. 109,921 .

4. Khan, M. I1.: Banc. Q.: Nizanuddin J. Agric: Foed Chem. 1995. 43. 2719

5. Racker. R.: Doring. K.: Reiser. O.J. Org. (Wem. 2000. 65. 6932.

6. A typical procedure for the synthesis of 3a. ta and 5a; A stirred solution of 1 a (496 me. 2.0 mmol). phenylenediamine (2a. 432 $\mathrm{mg} .4 .0 \mathrm{mmol})$ and $\mathrm{K}_{2} \mathrm{CO}_{3}(552 \mathrm{mg} .4 .0 \mathrm{mmol})$ in acetonitrile ( 10 mI.) was heated to retlux for $14 \mathrm{~h}$. Atter usual workup and column chromatographic separation (hexane ether. $3: 1$ ) allylic substitution products 3a-T: (304 mg. $\left.5 \mathrm{l}^{\circ}{ }_{0}\right)$ and 3a-7. (102 me. 17\%o) was obtained. P'ure $3 \mathbf{a}-E(296 \mathrm{mg} .1 .0 \mathrm{mmol})$ in acetic aicd $(3 \mathrm{~mL})$ was heated to $60-70^{\circ} \mathrm{C}$ during $18 \mathrm{~h}$. Alter usual workup and wolum chromatographic separation (hexanc ether. $3: 1-1: 2$ ) ta (9l me. $\left.36^{\circ} 0\right)$ and 5 a $\left(110 \mathrm{mg} .34^{\circ} \mathrm{o}\right)$ were isolated. $3 \mathrm{at}-E$ : oil: IR (KBr) $340.3,3.34,3246,1701 \mathrm{~cm}$ ': 'II NMR (CDCl 3 ) $\delta 1.34$ (t. $J-7.1$ Hz. $3 \mathrm{H}$ ). 3.50 (br s. $3 \mathrm{H}$ ). 4.10 (s. $2 \mathrm{H}$ ). 4.29 (๕. $J-7.1 \mathrm{H}$. $2 \mathrm{H}$ ). 6.55-6.78 (m. 4H). 7.33-7.46 (m. 5H). 7.91 (s. IH). ${ }^{1.7} \mathrm{C}$ NMR $\left(\mathrm{CDCl}_{3}\right) \& 14.27 .41 .38 .61 .10 .112 .95 .116 .19 .119 .36 .120 .23$. 128.64. 129.09. 129.50,129.73,134.82, 135.25. 136.97. 142.56, 167.78. 3a- $Z$ : oil: IR (KBr) $3404,3342,3246.1711 \mathrm{~cm}$ ': 'L NMR (CDCl) $\delta 1.11$ (t. $J-7.2 \mathrm{IJ} . .3 \mathrm{II}), 3.50(\mathrm{br} \mathrm{s} .3 \mathrm{II}), 4.10(\mathrm{~s}$, $2 \mathrm{H}) .4 .15$ (c. $J-7.2 \mathrm{~Hz} .2 \mathrm{H}) .6 .70-6.82(\mathrm{~m} .4 \mathrm{H}) .6 .89$ (s. $1 \mathrm{H}$ ). 7.24-7.30 (m. 5H): ${ }^{1.3} \mathrm{C}$ NMR $\left(\mathrm{CDCl}_{3}\right) \delta$ 13.74. 48.47. 60.82. 113.24 . 116.64. 119.48. 120.56. 127.99. 128.03. 128.32. 131.74. 1.34.77. 1.34.82. 1.35.61, 1.36.73, 168.76. ta: yellow solid, mp 155$157^{\circ} \mathrm{C}: \mathrm{IR}(\mathrm{KBr}) .3403,3354.3188,3058,1656.1625,138+\mathrm{cm}$ !: ${ }^{\mathrm{I}} \mathrm{I}$ NMR $\left(\mathrm{CDCl}_{3}\right) \delta 4.09$ (br s. 1H. NH). +.1 .3 (s. $2 \mathrm{H}$ ). 6.7.3-7.02 (m. 4H). 7.32-7.43 (m. 5H). 7.85 (s. lH). 8.76 (br s. $1 \mathrm{H} . \mathrm{NH}){ }^{13} \mathrm{C}$ NMR $\left(\mathrm{CLCl}_{3}\right) \delta 43.34 .118 .00 .119 .79 .120 .25 .123 .05 .126 .65$. $127.45\left(2 \mathrm{C}\right.$ by ${ }^{1} \mathrm{H}^{-1.2} \mathrm{C}$ hetero-COSY). 128.46. 130.97. 134.32. 1.37.03. 1.38.3i, 168.62: Mass (70 cV) $m z$ (rel intensit) $119(99)$ $1.34(20), 173(30), 221$ (34). 250 (M'. 100). 5a: oil: IR (KBr)

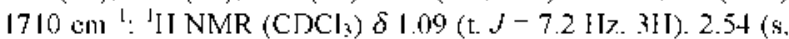
$3 \mathrm{H}) .4 .06(\mathrm{c} . J-7.2 \mathrm{~Hz} .2 \mathrm{H}) .5 .20$ (s. 2H). 7.02-7.64(m.9H). 8.01 (s. $1 \mathrm{H}):{ }^{12} \mathrm{C}$ NMR $\left(\mathrm{CDCl}_{3}\right) \dot{0} 13.89 .14 .21 .40 .43 .61 .24$. 109.98. 118.78. 121.54. 121.73. 127.89. 128.91. 129.17. 129.39. $1.34 .19 .135 .09,142.45,142.83,152.30,166.08$. 